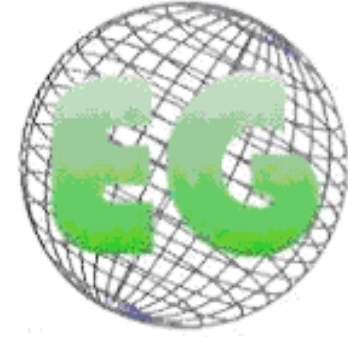

ISSN 1695-6141

\title{
La comunicación enfermera-cliente en el cuidado en las unidades de urgencias 24h: una interpretación en Travelbee
}

\author{
A comunicação enfermeira-cliente no cuidado em unidade de pronto atendimento 24h (UPA \\ 24h): uma interpretação em Travelbee \\ The communication nurse-patient in treatment at the emergency services 24 hours: an \\ interpretation in Travelbee
}

\author{
${ }^{*}$ Rocha Oliveira, Thalita **Faria Simões, Sonia Mara \\ *Máster en Enfermería/UFF. Enfermera obstétrica del Hospital Federal de Bonsucesso/Rio de Janeiro. \\ E-mail: oliveira.thalita@oi.com.br **Profesora Doctora de la Escuela de Enfermería Aurora de Afonso \\ Costa/UFF Brasil.
}

Palabras clave: enfermería; Atención de Enfermería; comunicación; Enfermería de Urgencia. Palavras-chave: enfermagem; cuidado de enfermagem; comunicação; Enfermagem em Emergência Keywords: Nursing; nursing service; communitacion; emergency nursing

\section{RESUMEN}

Investigación de carácter descriptivo y abordaje cualitativo que tiene por objetivo mostrar la comunicación enfermera-paciente en el cuidado de enfermería en las Unidades de urgencias $24 \mathrm{~h}$ y discutir los aspectos de la comunicación y de la relación interpersonal enfermera-paciente en el cuidado a la luz del referencial teórico de Joyce Travelbee. Los datos fueron obtenidos después de la apobación en el comité de ética a través de entrevista semi-estructurada aplicada a diez $2^{\circ}$ Tenientes Enfermeras Bombero Militar ubicado en el municipio de Belford Roxo. El análisis temático de las palabras posibilitó evidenciar tres categorías: la comunicación revelada en el trato de enfermería; la comunicación enfermera-paciente en los espacios del cuidado de la Unidad de Urgencias $24 \mathrm{~h}$ y los aspectos de la relación interpersonal enfermera-paciente.En resumen, por tratarse de una relación ser humano-ser humano la comunicación y el cuidado añadido en una Unidad de Urgencias ocurren en sus distintas posibilidades objetiva y afectiva, instrumental y expresiva, respectivamente, independiente del espacio del cuidado.

\section{RESUMO}

Pesquisa de caráter descritivo, abordagem qualitativa, visa caracterizar a comunicação enfermeirapaciente no cuidado de enfermagem em Unidade de Pronto Atendimento $24 \mathrm{~h}$ e discutir os aspectos da comunicação e da relação interpessoal enfermeira-paciente no cuidado à luz do referencial teórico de Joyce Travelbee. Os dados foram obtidos após aprovação no comitê de ética através de entrevista semi-estruturada aplicada à dez $2^{\circ}$ Tenentes Enfermeiras Bombeiro Militar neste cenário localizado no município de Belford Roxo. A análise temática das falas possibilitou evidenciar três categorias: a comunicação revelada no cuidado de enfermagem; a comunicação enfermeira-paciente nos espaços 
de cuidado da Unidade de Pronto Atendimento $24 \mathrm{~h}$ e aspectos da relação interpessoal enfermeirapaciente. Concluindo, por ser uma relação ser humano-ser humano, a comunicação e o cuidado implementados em uma Unidade de Pronto Atendimento 24h acontecem em suas faces objetiva e afetiva, instrumental e expressivo, respectivamente, independente do espaço de cuidado.

\section{ABSTRACT}

A descriptive survey and a qualitative boarding have as a goal to show the communication nurse-patient and the treatment in nursing that the $24 \mathrm{~h}$ emergency services and to discuss the aspects of the communication and the interpersonal relationship nurse-patient in the treatment regarding the theoretical reference by Joyce Travelbee. Data were obtained after having being approved in the committee of ethics through the semi-structured interview applied to ten 2nd Lieutenants Military Firewomen Nurses in the District of Belford Roxo. The thematic analysis of the words made possible to demonstrate three categories: the revealed communication in the treatment of nursing; the communication nurse-patient in the spaces of care of the24h Emergency Unit and the aspects of the interpersonal relationship nurse-patient. To sum up, due to the relation human being- human being the communication and the added care in an Emergency Unit occur in its different objective and affective possibilities, instrumental and expressive, respectively, independent of the space of care.

\section{INTRODUCCIÓN}

La comunicación forma parte de la historia del hombre en la sociedad y es fruto de la interacción social. Se trata de una relación entre emisor y receptor, entre signos, significados y códigos.

En enfermería es necesaria la comunicación verbal y no verbal, siendo una competencia o habilidad que posibilita el reconocimiento de las necesidades individuales, el establecimiento de una relación interpersonal significativa y el cuidado de enfermería. La percepción de señales corporales y de la lengua hablada representan la posibilidad de ir más allá de la ejecución de procedimentos técnicos al permitir que los sentimientos, las emociones y la historia de cada uno favorezcan la comprensión de la totalidad que este ser humano representa ${ }^{(1)}$.

La comunicación y la relación interpersonal enfermera-paciente son constituyentes de la enfermería. Al relacionase con el cliente, familia y comunidad, la enfermera marca su presencia profesional al expresar el cuidado dialógico(2).

Así, la comunicación es una de las herramientas básicas para sostener el proceso de "cuidar". Se revela como un eslabón esencial del cuidado al aproximar los sujetos, proporcionando momentos de intercambio, de interés y de preocupación con el otro ${ }^{(3)}$. Esto evidencia la interdependencia entre comunicación (a medio camino de la integralidad) y el cuidado (en el que la integralidad es esencialmente ella misma), toda vez que no hay acción de cuidar que no se constituya en una acción comunicativa ${ }^{(4)}$.

La integración entre comunicación y cuidado pasa por el cuidar expresivo por ser este cognitivo-afectivo e incentivador permitiendo al paciente luchar y utilizar sus sentidos a través de la relación persona-persona y sus lenguajes verbales y no verbales. EI cuidar por gestos y palabras permite al enfermero analizar e interpretar los mensajes para establecer la terapéutica de resolución de las necesidades afectadas ${ }^{(5)}$.

La importancia de la relación en el cuidado de enfermería está constituida por la esfera subjetiva cuando se refiere a sensibilidad, creatividad, intuición, vivencia, 
relación y expresiones afectivas. En la esfera objetiva hay desarrollo de técnicas, procedimientos y acciones instrumentales ${ }^{(6,7)}$.

Por tanto, en el momento del cuidado las dos caras se manifestan cuando la enfermera identifica lo que será necesario tales como condiciones materiales, equipamientos, condiciones naturales además de asegurarse de sus propias condiciones personales para realizar la acción. Esto incluye la disponibilidad, la acogida y el respeto. Este inicio de la acción de cuidar involucra la comunicación con el paciente por medio de palabras, gestos, postura y hasta incluso el silencio ${ }^{(8)}$.

Estos juicios permiten entender que la relación interpersonal enfermera-paciente atraviesa por matices de la comunicación y del cuidado: abarca el cuidado orientado para procedimientos, técnicas de fundamentos de enfermería y conocimiento científico (cuidado instrumental), lo que se presenta por una comunicación objetiva; e incluye el cuidado orientado a las cuestiones subjetivas, sentimentales y emocionales (cuidado expresivo) que se manifesta a través de una comunicación afectiva, la cual definimos por un lenguaje en que los sentimientos de los sujetos involucrados pasan por un intercambio de mensajes.

La comprensión de la acción comunicativa posibilitó observar durante el ejercicio profesional en Unidad de Urgencias $24 \mathrm{~h}$, que, al principio, el cuidado de enfermería está directamente articulado con el factor "tiempo". Esto porque ese escenario exige del profesional agilidad y dominio de procedimentos técnicos, cara a la inmediatez de resultados impuestos por situaciones de urgencias y emergencias, así como excesivo número de atenciones/día.

En emergencia, el tiempo tiene fundamental importancia en las relaciones establecidas, ya que por un lado el paciente exige que sus necesidades sean atendidas prontamente y por otro los profesionales precisan atender las demandas del paciente, ser rápido e inmediato como preconizan las normas de la institución ${ }^{(9)}$.

Considerando la importancia de la temática, se realizó una búsqueda sistematizada en periódicos de enfermería en el período de enero a marzo de 2009, con asociación de los descriptores comunicación y enfermería, en recorte temporal de 1998 a 2008. Tras sucesivas lecturas, se seleccionaron 40 artículos que explicitaban de forma amplia la temática comunicación ${ }^{(10)}$.

Los artículos trataban de la temática en varios escenarios de cuidado, como terapia intensiva, obstetricia, gerontología, por ello no identificamos aquel que abordase el contexto de cuidado en la emergencia. Aunque no había sido usado el descriptor emergencia, identificamos en la literatura científica un espacio de investigación a ser explorado. Hecho que despertó nuestro interés y promovió la curiosidad para el desarrollo de la disertación de mestrado en la Universidad Federal Fluminense, buscando la comprensión del fenómeno "comunicación enfermera-paciente en Urgencias 24h". Así, la investigación tuvo como objetivos caracterizar la comunicación enfermera-paciente en el cuidado de enfermería en Urgencias $24 \mathrm{~h}$ y discutir los aspectos de la comunicación y de la relación interpersonal enfermera-paciente en el cuidado en Urgencias $24 \mathrm{~h}$ a la luz de la teoría interaccionista de Joyce Travelbee.

La lectura de la obra "Intervención en enfermería psiquiátrica: el proceso de la relación de persona a persona" de la teórica de enfermería Joyce Travelbee, publicada en $1979^{(11)}$ evidenció la adherencia de los conceptos y presupuestos de la 
teoría interaccionista con el escenario de emergencia al aclarar que la enfermería es un proceso interpersonal y que la comunicación posibilita la expresión de sentimientos, valores y significados necesarios para que la enfermera cumpla uno de los objetivos de la relación persona-persona, que es el de ayudar a los individuos a enfrentar la experiencia de la enfermedad.

Además de los conceptos mencionados anteriormente, los siguientes presupuestos de la Teoria de Travelbee fundamentan esta investigación: la finalidad del cuidado se da por el establecimiento de la relación persona-persona en la medida en que toda acción de cuidar incluye relación interpersonal; la enfermera establece la relación a través de la comunicación para cumplir la finalidad de los cuidados, pues toda relación es permeada por aspectos verbales y no verbales y la enfermera ayuda a las personas a encontrar significado a la experiencia de la enfermedad ${ }^{(12)}$.

\section{METODOLOGÍA}

Estudio de carácter descriptivo, abordaje cualitativo, tuvo como escenario la Unidad de Urgencias 24h, localizada en el municipio de Belford Roxo. Un nuevo escenario político de cuidado, proyecto basado en la Política Nacional de Urgencia y Emergencia del Ministerio de Salud e implementado por la Secretaría de Salud y Defensa Civil del Estado de Rio de Janeiro en 2007.

Las Unidades de urgencias se estructuran por sectores: acogida, área central para escucha de las necesidades de los usuarios y orientar flujos; el sector de clasificación de riesgo, local para evaluar el grado de urgencia de las quejas de los pacientes lo que permite la priorización de la atención; el área amarilla para observación y estabilización de pacientes en condiciones críticas y semicríticas y el área roja destinada a la atención de pacientes graves, donde la tecnología es colocada como elemento esencial que compone el espacio.

Participaron como sujetos, todas las $2^{\circ}$ Tenientes Enfermeras Bombero Militar que trabajaban directamente con el cuidado de enfermería, constituyendo un total de diez entrevistadas.

La obtención de los testimonios ocurrió en los meses de febrero a abril de 2010 en dos momentos. En el primero se realizó la aproximación y ambientación como investigadora, con la intención de distanciar presupuestos construídos a partir de mi vivencia como enfermera Bombero Militar en Unidad de Urgencias 24h. En el segundo momento, de marzo a abril se aplicó la entrevista semi-estructurada constituída por la identificación de los sujetos en relación a la edad, año de conclusión de la graduación y cursos de especialización realizados. En seguida, se presentaron cinco cuestiones que buscaban entender cómo acontece la comunicación enfermera-paciente, el cuidado de enfermería y a relación interpersonal en la Unidad de Urgencias 24h.

Las entrevistas se realizaron respetando el día de turno de las declarantes. A fin de mantener el anonimato de las informaciones se determinó un nombre ficticio de identificación de las hablas a través de la asociación de la letra E de entrevista seguido por el orden numérico de las entrevistas, o sea, la primera entrevistada recibió la identificación E 01 y así en adelante.

Tras la transcripción completa, el análisis temático consistió en tres etapas: lectura fluctuante del conjunto de las comunicaciones; identificación de categorías por medio 
de expresiones o palabras significativas; proposición de inferencias y realización de interpretaciones, relacionándolas con el cuadro teórico seguido en la investigación ${ }^{(13)}$.

La investigación fue aprobada por el Comitê de Ética en Investigación del Hospital Universitario Antonio Pedro (CAEE $n^{\circ}$ 4432.0.000.258-09) a fin de cumplir lo que preceptúa la Resolución nº 196/96, del Consejo Nacional de Salud. La autorización de los sujetos del estudio para participación fue registrada a través del término de consentimiento libre y esclarecido, que fue presentado a las enfermeras previamente a la entrevista, así como los objetivos del estudio y la certificación de anonimato de las informaciones.

\section{RESULTADOS Y DISCUSIÓN}

Los datos revelaron que la mitad de las entrevistadas son adultos jóvenes en faja de edad de 20-29 años. En cuanto al año de conclusión de la graduación, la mayoría de las enfermeras militares se formó en los últimos cinco años y presenta diversidad de cursos de especialización como enfermería obstétrica, cuidado a pacientes críticos, enfermería del trabajo, entre otros.

El análisis temático de los testimonios permitió la constitución de tres categorías: la comunicación revelada em el cuidado de enfermería; la comunicación enfermerapaciente en los espacios de cuidado de la Unidad de Urgencias $24 \mathrm{~h}$ y aspectos de la relación interpersonal enfermera-paciente en la Unidad de Urgencias 24h.

\section{La comunicación revelada en el cuidado de enfermería}

Esta categoria reveló que la comunicación es percibida por la enfermera como esencialidad del cuidado de enfermería y de la interacción y como instrumento para alcanzar el sentimiento de confianza.

En la primera hay el reconocimiento de las enfermeras de que la comunicación y el diálogo son fundamentales para desarrollar una acción de cuidado y establecer la relación enfermera-paciente.

[...] en todos los momentos tiene que haber comunicación, no sólo a la hora de prestar el cuidado, sino antes incluso cuando el paciente está internado para interactuar con él (E 02).

La comunicación tiene que ser clara, tiene que ser precisa, tiene que ser directa y verdadera cuando la gente va a interactuar con ese paciente[...] yo creo que cuando ocurre de esta forma resulta todo más fácil, la gente consigue trabajar mejor (E 07).

La comunicación en sus aspectos verbales y no verbales es uno de los instrumentos básicos de la práctica de enfermería así como la destreza, la habilidad manual, el conocimiento científico, la observación, la creatividad, entre otros, pues permiten a la enfermera interactuar, relacionarse e implementar el cuidado de enfermería. La comunicación posibilita a la enfermera delimitar las metas de intervención y cumplir los objetivos de la enfermería como la oferta de ayuda. Y para ello, la enfermera debe dar énfasis a la habilidad de comunicarse y mantener una comunicación significativa. Así, la habilidad para comunicarse con otros seres humanos, de entender y ser entendido constituye una capacidad interpersonal esencial ${ }^{(11)}$. 
El desarrollo de habilidades en comunicación conduce también a una acción cuidativa amplia, ya que promueve el perfeccionamiento del saber-hacer profesional, colaborando con la calidad de las relaciones que se establecen ${ }^{(14)}$.

En el escenario de emergencia, la enfermera precisa estar atenta a las diferentes formas de comunicación no verbal, pues muchos pacientes que llegan a la unidad presentan condición clínica desfavorable para la comunicación verbal. La enfermera en ese momento necesita observar lo que la expresión facial y el cuerpo manifestan como señales y síntomas, a fin de evaluar el cuadro clínico objetivo y subjetivo e instituir el cuidado adecuado.

Así, una entrevistada indica que el cuidado surge a partir de la percepción y observación del otro, del conocimiento de las necesidades de quien procura ayuda y ello favorece que la enfermera esté atenta a las señales verbales y no verbales.

Para que yo implemente el cuidado eso el propio paciente lo va a demandar, su cuerpo va a hablar comigo [...] yo tambiém voy a entrevistarlo para saber lo que él necesita más y, sobre eso, trazar las conductas (E 05).

Cuando la enfermera establece la relación a través de la comunicación cumple la finalidad del cuidado ${ }^{(12)}$, pues en cualquier ambiente asistencial la comunicación verbal y no verbal están presentes, así como la interacción de los sujetos.

En este estudio, la comunicación es percibida por la enfermera como instrumento para alcanzar el sentimiento de confianza y de vínculo en la relación enfermerapaciente como se indica en las siguientes palabras.

Cuando usted comienza a desarrollar el diálogo con este paciente, usted pasa a ver que él pierde aquel grado de ansiedad, aquel recelo y pasa a tener más confianza en usted (E 01).

Yo creo que la integración entre comunicación y cuidado es usted atendiendo las necesidades sentidas y no sentidas por el paciente, o sea, el procedimiento técnico es el sentido y el no sentido en el cuidado sería estar cerca de él, saber si él tiene miedo, [...] creo que eso rompe una barrera y genera un poco má de confianza (E O3).

En general, en este ambiente los pacientes se encuentran tensos, temerosos y frágiles ante lo desconocido, la inesperada transición de un estado saludable a la proximidad con la muerte puede afectar el equilibrio emocional de pacientes y familias $^{(15)}$.

La aplicación de la teoría interaccionista en Unidad Neonatal posibilitó la observación de un cuidado de enfermería considerando la inclusión de los sujetos, el conocimiento del otro y la oferta de apoyo y confianza, lo que facilita la aproximación pacienteenfermera $^{(16)}$.

Transmitir confianza es expresión definida como el acto de ayudar al individuo a recuperar un estado de seguridad perdido. La enfermera que transmite confianza abre nuevos caminos de comunicación, es una persona sensible, sabe lo que decir y 
cuándo decirlo, se mantiene en silencio y utiliza formas no verbales para transmitir su interés. Además, la enfermera que se preocupa y hace todo lo posible para ayudar al enfermo es la persona que tiene mayor probabilidad de exhibir un comportamiento tranquilizador ${ }^{(11)}$.

La entrevistada destaca que reconocer al paciente como ser humano es percibirlo como alguien que precisa de ayuda, es ofrecer palabras de apoyo y aliento, incluso en un momento de dolor y sufrimiento por la enfermedad y hospitalización.

Porque usted está observando al paciente ante todo como ser humano, que precisa de su ayuda en aquel momento [...] y con palabras de apoyo y aliento [...] de que todo va a mejorar, ve que yo estoy haciendo esto por su bien aunque sea malo, es esencial para su mejoría (E 09).

En la teoría interaccionista, referente de este estudio, las enfermeras comprenden y acreditan el apoyo emocional y esto se debe, probablemente, no solo al hecho de ser sensitivas e intuitivas, sino también porque conocen el significado de esa actitud ante alguien que vivencia el dolor y el sufrimiento ${ }^{(11)}$. A través del apoyo emocional, la enfermera cumple uno de los presupuestos que es el de ayudar al enfermo a encontrar un sentido a su enfermedad ${ }^{(12)}$.

En el escenario de la Unidad de Urgencias $24 \mathrm{~h}$, estas actitudes están presentes en las acciones de la enfermera, facilitando el establecimiento del sentimiento de confianza y de apoyo emocional y que se traducen en el cuidado expresivo.

\section{La comunicación enfermera-paciente en los espacios de cuidado de la Unidad de Urgencias $24 \mathrm{~h}$}

Esta categoria expresa la comunicación como escucha, aproximación y direccionamiento del cuidado, así como intervención y sensibilidad.

La comunicación expresada como escucha, aproximación y direccionamiento del cuidado se caracteriza por el énfasis en orientaciones/informaciones, considera la privacidad y señala el retraimiento personal de la enfermera.

[...] yo creo esencial al enfermero presente en el recibimiento, porque el enfermero va a dar el toque de conocimiento, orientando al paciente sobre lo que él va a hacer, lo que le puede ocurir (E 01).

[...] en el recibimiento la comunicación que yo percibí era un buen día señor, qué es lo que pasó, cuál es su queja [...] dependiendo de la queja usted preguntaba más cosas [...] y ahí usted intentaba ser acogedor (E 06).

El recibimiento de la Unidad de Urgencias $24 \mathrm{~h}$ es el espacio de cuidado donde el usuario del sistema de salud tiene el primero contacto con el servicio. En el local siempre están presentes una enfermera y un técnico de enfermería que reciben a los pacientes identificando el nombre y el porqué de acudir a la unidad. En este momento de aproximación enfermera-paciente, la comunicación se expresa en informaciones/orientaciones relacionadas con la educación de salud y sobre el flujo de la unidad una vez que las Unidades de Urgencias $24 \mathrm{~h}$ se configuran como nueva propuesta de atención de emergencia. 
El sentido de la palabra acoger se revela cuando la enfermera dispensa atención en la relación, da crédito al ser humano, ejercita la escucha de las quejas y necesidades do paciente y las valora, instituyendo en su práctica una herramienta relacional ${ }^{(17)}$.

La escucha comienza a darse a partir del momento en que el profesional echa mano del conocimiento técnico-científico y se dispone a escuchar al otro lo que proporciona una mejor atención individual y una mayor capacidad de acogida por parte de la institución de salud ${ }^{(4)}$.

Posterior a la acogida del usuario es el direccionamiento para la atención en una de las especialidades de clínica médica, pediatría u odontología.

En la clasificación de riesgo, la enfermera realiza la consulta de enfermería dirigiendo la comunicación inicialmente para rellenar un formulario, a fin de observar factores de riesgo y evaluar la gravedad de los síntomas basada en el análisis de protocolos preeestablecidos que priorizan y agilizan la atención para la consulta médica.

[...] en la clasificación de riesgo usted va a tener más un contacto viendo lo que el paciente presenta, los factores de riesgo, usted va a explicar nuevamente cómo funciona el local, la estructura (E 08).

En esta fase, la comunicación se presenta como un instrumento necesario para la metodología de la asistencia, principalmente en lo que se refiere al histórico de enfermería, una vez que la colecta de datos incluye la identificación de las fuentes de datos que son pacientes o familias, la adquisición de informaciones y la organización y registro de los datos. Consiste en una comunicación interpersonal y exige del enfermero la habilidad para obtener datos relevantes ${ }^{(18)}$.

Las entrevistadas indican que en los espacios de recepción y de clasificación de riesgo, la comunicación enfermera-paciente tiende a ser objetiva debido a la agilidad que se da en emergencia, así como para promover la fluidez de la atención, evidenciando el modelo de comunicación informativo, cuando un individuo solicita una información, da instrucciones y órdenes a los demás ${ }^{(11)}$.

Aunque el modelo de comunicación informativo sea posible en los diversos microambientes constituyentes de una Unidad de urgencias $24 \mathrm{~h}$, el proceso de comunicación atraviesa otras dimensiones tales como actitudes de comprensión, escucha, disposición, ayuda, o sea, de acogida, como revela la entrevista siguiente.

Ah! En la clasificación de riesgo [...] nuestra explicación de que la gente está allí para ver la gravedad de la situación, saber la biografía de la persona y a veces la persona lloraba, se desahogaba, a veces la gente es mucho más que enfermero, es oyente, psicólogo es mucho más (E 09).

La privacidad proporcionada en el sector de clasificación de riesgo por ser ambiente cerrado puede favorecer la comunicación afectiva enfermera-paciente a través de la aproximación de los sujetos, atención y escucha sensible. Posibilita también que cuestiones íntimas y subjetivas constituyentes del ser humano sean abordadas en el momento de la consulta de enfermería, valorizando la relación sujeto-sujeto. 
Usted conseguía comunicarse mejor porque usted estaba sentado a la misma altura que el paciente, estaban ambos sentados en una sala, tenía privacidad, usted hacía más preguntas, usted recogía más informaciones [...] muchas veces se sentaban personas que querían discurrir sobre sus vidas, contar una historia enorme (E 06).

Otro aspecto de la comunicación enfermera-paciente en Unidad de Urgencias 24h expresada por los sentimientos fue la comunicación resultante de retraimiento personal de la Enfermera. En general, las enfermeras presentam características de cordialidad, de educación y de disposición a escuchar las quejas de los pacientes en la acogida. Por ello, la población al encontrarse con una situación de insatisfacción en la atención siente la necesidad de manifestarse a través de reclamaciones.

Para el paciente su condición física y ansiedad es lo que determina la organización de la atención. De esta forma, la angustia generada por la espera de la atención hace que él observe cada paso de los profesionales de salud, cuestionando a veces de forma hasta agresiva cuando perciben que alguien está siendo atendido en su frente ${ }^{(19)}$.

Esto genera desgaste en la comunicación y en la relación establecida en este espacio de cuidado y la comunicación asume característica de retraimiento.

[...] En vez de procurar saber lo que realmente estaba sucediendo, nos culpaban porque la atención no era completa y quedaba en aquella cuestión conflictiva y, a veces, desgastante allá (en la acogida) (E 07).

Lo que sucede antes de la interacción puede determinar la disposición que tiene el emisor y el receptor de aceptar y escuchar el mensaje y la forma cómo el mensaje fue emitido ${ }^{(11)}$. Así, cuando la enfermera se ve ante una situación de enfrentamiento delante del paciente surge la dificultad en establecer comunicación adecuada por el aflujo de sentimientos diferenciados, expresando una actitud más fría, más impersonal y de autocontrol.

Esto porque el ser humano no es capaz de aceptar a toda persona que conoce automáticamente. Intelectualmente, la enfermera puede comprender por qué la persona enferma posee ciertas actitudes, mas al vivenciar una experiencia de no apertura del paciente es posible su no aceptación ${ }^{(11)}$.

De esta forma, las participantes del estudio revelan que en el cuidado en Unidad de Urgencias $24 \mathrm{~h}$ acaban asumiendo actitud de retraimiento personal en detrimento de ser profesionales y militares. En esta situación acontece el silencio.

[...] yo intento controlarme al máximo para tener más paciencia con los pacientes, porque ellos tienen un montón de problemas y juzgan todo por encima de usted. Yo intento tener autocontrol. Se precisa mantener una postura hasta porque la gente es militar (E 04).

La comunicación enfermera-paciente considerada como intervención y sensibilidad sucede por la demanda de trabajo y alta rotatividad presente en los espacios de cuidado sala amarilla y sala roja de una Unidad de Urgencias $24 \mathrm{~h}$. 
[...] en la sala amarilla la comunicación queda un poco perjudicada hasta por la demanda de trabajo (E 01).

[...] ella (la comunicación) tiene que ser mejorada en el sentido de que falta tiempo, mucho servicio para dar la orientación debida, mucha sobrecarga de trabajo, tipo muchos pacientes, usted no puede dar todas las orientaciones (E 10).

La sala amarilla de una Unidad de Urgencias $24 \mathrm{~h}$ se caracteriza por ser área destinada a la estabilización de pacientes críticos, semicríticos y aquellos que precisan de observación. La dinámica de funcionamiento asociada a la alta demanda y alegación de falta de tiempo de los profesionales dirigen la comunicación enfermerapaciente a la objetividad, en la medida en que su atención está centrada en las quejas clínicas, en las necesidades humanas básicas y en la prestación del cuidado instrumental.

Este escenario, sumado a las características de los profesionales y a la propia atención de emergencia, acciones rápidas y precisas, conjuntamente con la vivencia del sufrimiento de pacientes y familiares tiende a generar una comunicación del enfermero de forma impersonal y mecanizada, limitando al profesional al cumplimiento de su papel instrumental( ${ }^{(20)}$. Esto muestra que en diversos momentos el cuidado en la unidad de emergencia posee características mucho más técnicas que expresivas $^{(21)}$.

La objetividad en la comunicación puede ser definida como la capacidad de la enfermera en observar lo que realmente está aconteciendo con el paciente, sin dejarse influenciar por sentimientos subjetivos, situación en la cual asume una posición imparcial ${ }^{(11)}$.

Por ello, cuando se alarga el periodo de hospitalización o cuando cesa la situación de urgencia, la enfermera dirige su atención a cuestiones que van más allá de la objetividad, de lo biológico. Ella ejerce una comunicación valorando la dimensión afectiva e implementa el cuidado expresivo.

En la sala amarilla los pacientes quedan como en un espacio de cuidado, yo creo que usted acaba acogiendo más, relacionándose más y queriendo saber el porqué de que él esté en aquella situación; mas si fuera muy rotativo, sólo entrar y estabilizar el cuadro, yo creo que la comunicación es aquella cosa [...] procedimiento, técnico [...], mas cuando el paciente permanece por más de dos días usted ya consigue ver si hay alguna carencia (E O3).

Otro espacio de cuidado de la Unidad de Urgencias 24h, la sala roja, se asemeja a una unidad de tratamiento semiintensivo y se destina a la recepción y permanencia de pacientes en cuadros graves donde la tecnología predomina en la intervención y recuperación de vidas.

En la sala roja la comunicación queda comprometida, cuando el paciente va para allá, él está en estado grave, usted no puede establecer una comunicación con un paciente sedado, solo una comunicación entre los profesionales que allí actúan, procurando enfatizar la medida en que este profesional tiene que estar preparado para agilizar la atención (E 01). 
El paciente de la sala roja está incomunicado, su patología es de mayor gravedad y la gente prácticamente no tiene una comunicación en el primer momento con ese paciente, todo es correr y estabilizar su cuadro de origen (E 07).

La comunicación enfermera-paciente en la sala roja revela su cara objetiva asociada al cuidado instrumental, una vez que es instituido por la sistematización institucional que el foco de los profesionales debe estar en intervenir y recuperar vidas, a través del uso de la tecnología y procedimientos técnicos.

La emergencia posee aspectos peculiares por ofrecer servicios de alta complejidad, por la busca de estabilización de las condiciones vitales del paciente exigiendo agilidad y objetividad en las acciones de los profesionales que allí actúan ${ }^{(15)}$.

Tal aspecto es expresado en las entrevistas al explicitar que la preocupación de los profesionales que allí actúan está dirigida para la técnica y estabilización del cuadro de la víctima. Resalta la comunicación entre el equipo de salud como instrumento que posibilita acciones realizadas de forma rápida, inmediata y de efectividad en la atención.

Por eso, tras alcanzar la estabilización o mejora del cuadro clínico del paciente o hasta su derivación a otra institución hospitalaria, la enfermera deja fluir la cara diferenciada de la comunicación y del cuidado al demostrar la preocupación de informar incluso a los pacientes graves, entubados y sedados lo que será realizado durante el cuidado instrumental.

Siempre procuramos avisar al paciente cuando vamos a hacer alguna cosa más traumática: joye! Voy a aspirar, incluso estando sedado, la gente sabe que la audición es una de las cosas de que la gente tiene control mientras aún tiene vida (E 05).

[...] yo me controlaba mucho en la sala roja, incluso con el paciente sedado yo me comunicaba con él, no sólo por el tacto, mas también de llegar y decir mi nombre y decir lo que yo iba a hacer (E 06).

La comunicación no verbal asume relevancia en estas situaciones a través de la observación de la enfermera de las señales emitidas por el cuerpo del paciente, cuando siente dolor, entre otros.

De esta forma, aunque la tecnología sea elemento esencial del cuidado en la sala roja, se percibe que el respeto por el otro y la sensibilidad revelan la comunicación afectiva y el cuidado expresivo implementados por la enfermera de la Unidad de urgencias $24 \mathrm{~h}$.

\section{Aspectos de la relación interpersonal enfermera-paciente en la Unidad de Urgencias 24h}

Esta categoría se relaciona con las actitudes constituyentes de las enfermeras en la relación interpersonal en una Unidad de urgencias 24h. Aquellas que significan la relación interpersonal son expresadas en estrategias y sentimientos de la enfermera para que una relación interpersonal se establezca. 
[...] usted se coloca en el lugar del paciente para evaluar qué necesidades tiene, procurar tener aquella conducta que usted querría para usted (E 01).

Yo creo que usted tiene que estar siempre solícito cuando el paciente te llama (E 04).

[...] usted tiene que estar conversando todo el tiempo con el paciente [...] observando ese paciente [...] es el contacto directo todo el tiempo (E 08).

Las enfermeras demuestran que la relación interpersonal con el paciente debe ser permeada por actitudes como colocarse en el lugar del otro, tener disponibilidad para oir, para conversar, ser solícita, atenciosa y cordial, tener una actitud de oferta de ayuda, mantener contacto directo y demostrar preocupación con el paciente.

Estas actitudes posibles de las enfermeras evidenciadas en los testimonios indican una comunicación afectiva, constituyente del cuidado expresivo que traspasa la relación interpersonal.

En consonancia con los resultados de este estudio se encontraron otros estudios. En un ambiente de cuidado a paciente crítico se identificó la importancia de valorar sentimientos presentes en el ser humano como la afectividad, la sensibilidad, la empatía y la participación emocional descritos en la teoría de la relación personapersona ${ }^{(22)}$. Otro estudio señala que el profesional de enfermería debe ejercer además de competencia técnica, actitudes como escucha, dedicación al otro y comunicación ${ }^{(23)}$.

Estos comportamientos de la enfermera participan la existencia del compromiso emocional en la relación, o sea, la capacidad de transcender a sí mismo e interesarse por la otra persona, a través de la capacidad de percibir a los otros como seres humanos únicos y de expresar las emociones y sentimientos en la interacción con el paciente ${ }^{(11)}$.

Así, el fenómeno cuidar se revela a través de un proceso comunicativo e interactivo que exige dedicación y compromiso de aquel que cuida y de aquel que es cuidado. Se confirma así un presupuesto de la Teoría Interaccionista de que la finalidad del cuidado se da por el establecimiento de la relación persona-persona ${ }^{(12)}$.

En cuanto a las actitudes de la enfermera que objetivan la relación interpersonal en la Unidad de Urgencias 24h, estas expresan la objetividad/superficialidad una vez que en el cotidiano del cuidado no valoran la aproximación y mantenimiento del diálogo con el paciente.

[...] hablando sinceramente yo no valoro mucho eso de permanecer al borde de la cama conversando con el paciente [...] es hasta una defensa [...] justamente para evitar su mayor contacto (E 05).

[...] porque las personas acaban cayendo en un sistema de defensa personal y hacen las cosas medio mecanizadas (E 06).

En general las actitudes de las enfermeras se manifiestan por actuar en un contexto intenso de cuidado, donde frecuentemente, las emociones de los sujetos involucrados están afloradas. En este sentido, a pesar de la superficialidad en la relación interpersonal, entendiendo no ser esta constituyente de la enfermera, pero sí una 
expresión impuesta por el escenario de cuidado en situaciones en las cuales la afectividad está del lado de la racionalidad en el cuidado y de la relación interpersonal.

\section{CONSIDERACIONES FINALES}

El estudio reveló que las enfermeras perciben y entienden la comunicacióno verbal y no verbal como elemento clave no solamente para desarrollar el cuidado en sus aspectos técnicos, sino también para promover la relación enfermera-paciente. Demuestran que la oferta de palabras de confort, tranquilidad y apoyo emocional favorecen el sentimiento de confianza explicitado en la Teoría Interaccionista y la formación del vínculo enfermera-paciente.

El análisis de las entrevistas indica características de la comunicación y del cuidado en cada espacio de cuidado de una Unidad de Urgencias $24 \mathrm{~h}$. En el recibimiento y en la clasificación de riesgo, a pesar de que la comunicación enfermera-paciente se presenta objetiva por la necesidad de orientación y direcionamiento de flujo, se evidencian otras actitudes. En el recibimiento, la postura de la enfermera está pautada en la escucha sensible, en la valorización de las quejas, lo que representa el sentido de la palabra acoger.

Ya en la clasificação de riesgo, la comunicación se orienta a la cumplimentación del instrumento de clasificación de riesgo y al abordaje de necesidades subjetivas que se dan en el proceso-salud enfermedad. Otra singularidad se refiere al primer momento de la interacción, en el que la enfermera es cordial y solícita, mas cuando ocurre la insatisfacción del paciente ella precisa mantener autocontrol y retraimiento personal.

En los espacios de cuidado de las salas amarilla y roja, la comunicación se presentó en su objetividad expresada en el énfasis del cuidado instrumental, por ser, respectivamente, sectores con propósito de estabilización de pacientes críticos y semicríticos y de intervención y recuperación de vidas. Sin embargo, las enfermeras no dejaron de manifestar la sensibilidad en el cuidado a través de la comunicación verbal y no verbal.

En este sentido, los resultados del presente estudio evidenciaron características de la comunicación y del cuidado de enfermería en cada espacio de cuidado. Ora la comunicación enfermera-paciente se presenta objetiva por las especificidades del escenario Unidad de Urgencias $24 \mathrm{~h}$, ora la comunicación es afectiva representada por el aflorar de la sensibilidad, del respeto, de la escucha activa, del estar disponible y de la oferta de ayuda que representa el compromiso emocional en la relación interpersonal enfermera-paciente descrito en la teoría interaccionista de Joyce Travelbee.

De esta forma, esta investigación aporta contribuciones al elucidar la comunicación enfermera-paciente en el cuidado de enfermería y las relaciones interpersonales bajo la óptica de una teoría interaccionista aplicada en un nuevo escenario político nacional, la Unidad de Urgencias 24h. Sin embargo, no agota la temática, y sí incentiva reflexiones y otras investigaciones en la práctica asistencial de la enfermería. 


\section{BIBLIOGRAFIA}

1. Souza LNA, Padilha MICS. A comunicação e o processo de trabalho em enfermagem. Texto Contexto Enferm. 2002 jan-abr; 11(1):11-30.

2. Simões SMF. O significado do pensar/fazer da prática do enfermeiro: uma revisão sistemática de artigos da REBEn 1932-1971. Esc Anna Nery Rev Enferm. 2007 set; 11(3): 509-14.

3. Baggio MA, Callegaro GD, Erdmann AL. Compreendendo as dimensões de cuidado em uma unidade de emergência hospitalar. Rev Bras Enferm. 2008 set-out; 61(5):552-7.

4. Xavier C, Guimarães C. Uma semiótica da integralidade: o signo da integralidade e o papel da comunicação. In: Pinheiro R, Mattos RA. Cuidado: as fronteiras da integralidade. Rio de Janeiro (RJ): Hucitec; 2004. p.133-155.

5. Coelho MJ. Maneiras de cuidar em enfermagem. Rev Bras Enferm. 2006 nov-dez; 59(6):745-51.

6. Figueiredo NMA de, Machado WCA, Porto IS. Dama de branco, Dama de negro: o cuidado na fronteira vida / morte. Rev Enferm UERJ.1995; 3(2):139-49.

7. Teixeira ER. A crítica e a sensibilidade no processo de cuidar na enfermagem. Esc Anna Nery Rev Enferm. 2004 dez; 8(3): 361-9.

8. Waldol VR. Momento de cuidar: momento de reflexão na ação. Rev Bras Enferm. 2009 jan-fev; 62(1): 140-5.

9. Baradel A. Os minutos da comunicação no serviço de emergência. In: Silva MJP, organizadora. Qual o tempo do cuidado? Humanizando os cuidados de enfermagem. São Paulo (SP): Loyola; 2004. p.29-37.

10. Oliveira TR, Simões SM. Communication faces in the practice of nursing. A bibliographic research. Online Brazilian Journal of Nursing [periódico na internet]. 2010; [Citado 2011 Mar 20]; 9(2) [aprox. 9 telas]. Disponível em:

http://www.objnursing.uff.br/index.php/nursing/article/view/3116

11. Travelbee J. Intervención en enfermeria psiquiatrica: el proceso de la relacion de persona a persona. Colombia: Organizacion Mundial de la Salud; 1979.

12. Leopardi MT. Teoria e método em assistência de enfermagem. $2^{\circ} \mathrm{ed}$. Florianópolis (SC): Soldasoft; 2006.

13. Minayo MCS. O desafio do conhecimento: Pesquisa Qualitativa em Saúde. São Paulo (SP): Hucitec; 2007.

14. Bites Júnior A, Matheus MCC. Comunicação. In: Cianciarullo TI. Instrumentos básicos para o cuidar: um desafio para a qualidade da assistência. $1^{\circ} \mathrm{ed}$. Belo Horizonte (MG): Atheneu; 2007. p.61-73.

15. Dal Pai D, Lautert L. Suporte humanizado no Pronto Socorro: um desafio para a enfermagem. Rev Bras Enferm. 2005 mar; 58(2): 231-4.

16. Oliveira MMC, Almeida $\mathrm{CB}$, Araújo TL, Galvão MTG. Aplicação do processo de relação interpessoal de Travelbee com mãe de recém-nascido internado em uma unidade neonatal. Rev Esc Enferm USP. 2005; 39(4): 430-6.

17. Ministério da Saúde (BR). Acolhimento com classificação de risco nos serviços de urgência. Brasília (DF); 2009.

18. Stefanelli MC, Carvalho EC de, organizadoras. A comunicação nos diferentes contextos da enfermagem. Baurueri (SP): Manole; 2005.

19. Gatti MFZ. O tempo urgente dos protagonistas do serviço de emergência. In: Silva MJP da, organizadora. Qual o tempo do cuidado? Humanizando os cuidados de enfermagem. São Paulo (SP): Loyola; 2004. p. 29-37.

20. Souza RB de, Silva MJP da, Nori A. Pronto- Socorrro: uma visão sobre a interação entre profissionais de enfermagem e pacientes. Rev. Gaúcha Enferm. 2007; 28(2): 242-9. 
21. Silva AL da, Nascimento KC do, Virgílio MS, Mendonça RS. Análise dos fatores de cuidado de Watson em uma unidade de emergência. Rev. Gaúcha Enferm. 2002 jul; 23(2): 27-50.

22. Silveira RS da, Lunardi VL, Lunardi Filho WD, Oliveira AMN de. Uma tentativa de humanizar a relação da equipe de enfermagem com a família de pacientes internados na UTI. Texto Contexto Enferm. 2005; 14(esp):125-30.

23. Chistóforo BEB, Zagonel IPS, Carvalho DS. Relacionamento enfermeiro-paciente no pré-operatório: uma reflexão à luz da teoria de Joyce Travelbee. Cogitare Enferm. 2006 jan-abr;11(1):55-60. 